\title{
The principle of maximum entropy explains the cores observed in the mass distribution of dwarf galaxies
}

\author{
Jorge Sánchez Almeida ${ }^{1,2}$, Ignacio Trujillo ${ }^{1,2}$, and Angel Ricardo Plastino ${ }^{3}$ \\ ${ }^{1}$ Instituto de Astrofísica de Canarias, La Laguna, Tenerife 38200, Spain \\ e-mail: jos@iac.es \\ 2 Departamento de Astrofísica, Universidad de La Laguna, La Laguna, Spain \\ 3 CeBio y Departamento de Ciencias Básicas, Universidad Nacional del Noroeste de la Prov. de Buenos Aires, UNNOBA, CONICET, \\ Roque Saenz Peña 456, Junin, Argentina
}

Received 15 August 2020 / Accepted 17 September 2020

\begin{abstract}
Cold dark matter (CDM) simulations predict a central cusp in the mass distribution of galaxies. This prediction is in stark contrast with observations of dwarf galaxies that show a central plateau or "core" in their density distribution. The proposed solutions to this core-cusp problem can be classified into two types. One invokes feedback mechanisms produced by the baryonic component of the galaxies and the other assumes that the properties of the dark matter particle depart from the CDM hypothesis. Here we propose an alternative yet complementary explanation. We argue that cores are unavoidable in the self-gravitating systems of maximum entropy that result from non-extensive statistical mechanics. Their structure follows from the Tsallis entropy, which is attributed to systems with long-range interactions. Strikingly, the mass density profiles predicted by such thermodynamic equilibrium match the observed cores without any adjustment or tuning. Thus, the principle of maximum Tsallis entropy explains the presence of cores in dwarf galaxies.
\end{abstract}

Key words. gravitation - galaxies: dwarf - galaxies: fundamental parameters - galaxies: structure - dark matter

\section{Introduction}

The total mass density of low-mass galaxies flattens up at their center, showing what is known as a core. This observational fact has been noted as a long-standing problem of the $\Lambda$ CDM paradigm (e.g., see recent reviews by Weinberg et al. 2015 and Del Popolo \& Le Delliou 2017) since early dark-matteronly numerical simulations predicted the existence of density cusps, rather than cores, in the inner regions of galaxies Moore (1994). A popular explanation of the so-called "core-cusp problem" relies on the inclusion of baryon physics in the simulations, which, through gravity, couples baryon processes with dark matter (DM). Explosive baryon-driven events at the center of the galaxies produce sudden changes in the gravitational potential which, integrated over time, turn the DM distribution from cusp to core (Governato et al. 2010). Alternatively, the core-cusp problem may also point out a failure of the cold DM hypothesis (Weinberg et al. 2015; Del Popolo \& Le Delliou 2017). Solutions include considering warm DM, whose freestreaming velocities erase primordial fluctuations on small scales (Colín et al. 2000), or by assuming self-interacting DM, where the scattering between DM particles redistributes energy and momentum generating inner cores (Spergel \& Steinhardt 2000).

Here, we propose an alternative solution to the core-cusp problem based on the principle of maximum Tsallis entropy and the polytropes it leads to. For theoretical reasons (presented in Sect. 2) polytropes may provide a good representation for the distribution of mass within galaxies and they all have cores. Therefore, the question arises as to whether the cores of the polytropes reproduce the cores observed in the matter distribution of dwarf galaxies. Here, we show that they do so without any free parameter (Sect. 3). Polytropes describe thermodynamic (or meta-stable) equilibrium configurations of self-gravitating systems under special conditions. Thus, our result suggests that these conditions are met in dwarf galaxies and may drive their internal structure (Sect. 4).

\section{Maximum-entropy self-gravitating systems and polytropes}

Galaxies are self-gravitating structures which, among all possible equilibrium configurations, end up having only those that are consistent with a stellar mass surface density profile resembling a Sérsic function (e.g., Blanton et al. 2003; van der Wel et al. 2012) ${ }^{1}$. Their settling into this particular configuration could be due either to some fundamental physical process (as it happens with the velocities of the molecules in a gas) or to the initial conditions that had given rise to the system (Binney \& Tremaine 2008). The mass distribution in galaxies is currently explained as the outcome of the initial conditions (Cen 2014; Nipoti 2015; Ludlow \& Angulo 2017; Brown et al. 2020). The option of a fundamental process determining the configuration is traditionally discredited because, in following the principles of

1 The Sérsic functions include exponential disks, observed in dwarf galaxies (e.g., de Jong \& van der Kruit 1994), and de Vaucouleurs 1/4-profiles, characteristic of massive ellipticals (e.g., de Vaucouleurs 1948). 
statistical physics, it should correspond to the most probable configuration of a self-gravitating system and, thus, it should result from maximizing the entropy. The use of the classical Boltzmann-Gibbs entropy leads to a distribution with infinity mass and energy (Binney \& Tremaine 2008; Padmanabhan 2008), disfavoring this explanation. In the standard BoltzmannGibbs approach, however, the long-range forces that govern self-gravitating systems are not properly taken into account. Systems with long-range interactions admit long-lasting metastable states described by a maximum entropy formalism based on Tsallis $\left(S_{q}\right)$ non-additive entropies (Tsallis 1988, 2009, and references therein). Observational evidence for the $S_{q}$ statistics has been found in connection with various astrophysical problems (Livadiotis \& McComas 2013; Silva et al. 2013). In particular, the maximization under suitable constraints of the Tsallis entropy of a Newtonian self-gravitating $N$-body system leads to a polytropic distributions (Plastino \& Plastino 1993; Lima \& de Souza 2005), which has finite mass and a shape closely resembling the DM distribution found in numerical simulations of galaxy formation (Navarro et al. 2004; Calvo et al. 2009). In the current cosmological model, DM provides most of the gravitational pull needed for the ordinary matter to collapse and form visible galaxies, and thus, polytropes can approximately describe the gravitational potential of galaxies. As we show below, the mass density associated with a polytrope always has a core. The question arises as to whether the cores of the polytropes reproduce the cores observed in the matter distribution of dwarf galaxies, thus providing an alternative view for solving the core-cusp problem (Sect. 1).

A polytrope of index $m$ is defined as the sphericallysymmetric self-gravitating structure resulting from the solution of the Lane-Emden equation for the (normalized) gravitational potential $\psi$ (Chandrasekhar 1967; Binney \& Tremaine 2008),

$\frac{1}{s^{2}} \frac{\mathrm{d}}{\mathrm{d} s}\left(s^{2} \frac{\mathrm{d} \psi}{\mathrm{d} s}\right)= \begin{cases}-3 \psi^{m} & \psi>0 \\ 0 & \psi \leq 0 .\end{cases}$

The symbol $s$ stands for the scaled radial distance in the 3D space and the mass volume density is reco vered from $\psi$ as

$\rho(r)=\rho(0) \psi(s)^{m}$,

$r=b s$,

where $r$ stands for the physical radial distance and $\rho(0)$ and $b$ are two arbitrary constants. Equation (1) is solved under the initial conditions $\psi(0)=1$ and $\mathrm{d} \psi(0) / \mathrm{d} s=0^{2}$. Figure 1 illustrates the variety of physically admissible polytropes, with the range of polytropic indexes

$3 / 2 \leq m \leq 5$,

set because polytropes with $m \leq 3 / 2$ are unstable or have infinite density and those with $m>5$ have infinite mass (Plastino \& Plastino 1993; Binney \& Tremaine 2008). In order to compute the polytropes in Fig. 1, Eq. (1) was split into a system of two first-order differential equations for $\psi$ and $\mathrm{d} \psi / \mathrm{d} s$, which were integrated from $s=0$ using Lsoda (Hindmarsh 2019), as implemented in python (scipy.odeint).

We note that all polytropes have cores, in the sense that $\mathrm{d} \ln \rho / \mathrm{d} \ln r \rightarrow 0$ when $r \rightarrow 0$. This property follows from the initial condition $\mathrm{d} \psi(0) / \mathrm{d} s=0$ and Eq. (2). It is shown by the density profiles displayed in Fig. 1.

\footnotetext{
2 Equation (1) also admits solutions with $\mathrm{d} \psi(0) / \mathrm{d} s \neq 0$, but those are discarded because they have infinite central density and total mass (e.g., Binney \& Tremaine 2008).
}

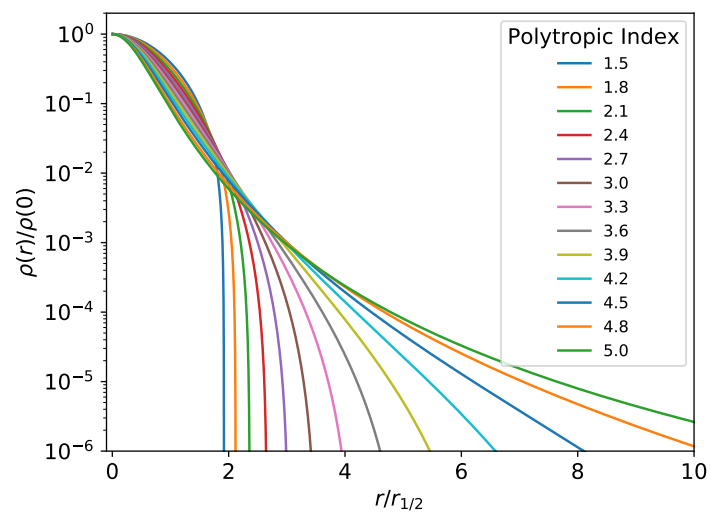

Fig. 1. Mass volume density resulting from the numerical solutions of the Lane-Emden equation (polytropes). Polytropes are self-gravitating systems having maximum Tsallis entropy. The curves are normalized to the central density and to the half-mass radius $\left(r_{1 / 2}\right)$. The examples show the range of physically plausible solutions, with the corresponding polytropic index given in the inset.

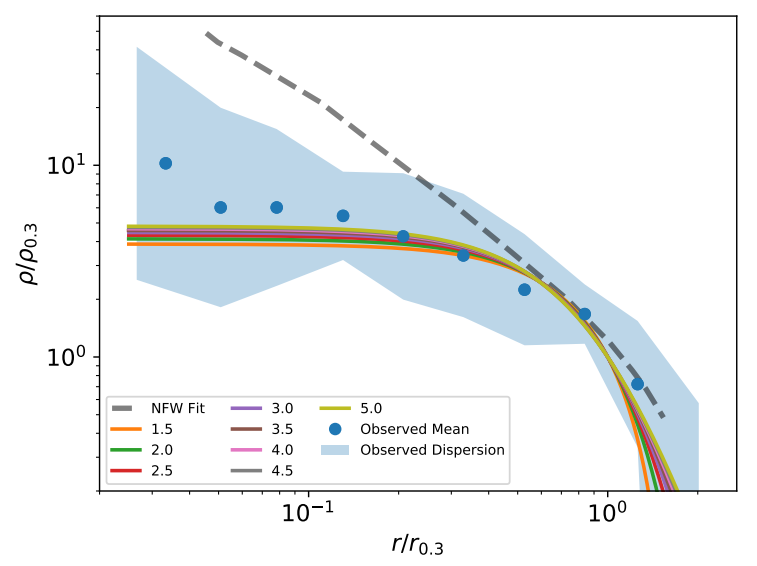

Fig. 2. Density profile observed in the inner regions of the 26 "Little Things" galaxies from Oh et al. (2015) (blue symbols and blue region give the mean and the root mean square (RMS) dispersion among the different objects). To reduce scatter, the observed densities and radii are normalized to the density and radius where the logarithmic derivative of the circular velocity equals $0.3\left(\mathrm{~d} \log v_{c} / \mathrm{d} \log r=0.3\right)$, denoted as $\rho_{0.3}$ and $r_{0.3}$, respectively. Polytropes are parameter-free in this representation (solid lines with the corresponding indexes given in the inset). The dashed line gives a best-fit to the observed density using a NavarroFrenk-White (NFW) profile (Oh et al. 2015), which does not follow the observed core.

\section{Results}

Figure 2 shows the state-of-the-art observation of galaxy cores in dwarf galaxies by Oh et al. (2015), which is based on 26 galaxies with stellar masses of $6.5 \leq \log \left(M_{\star} / M_{\odot}\right) \leq 8.2$ (blue symbols with the blue region giving the RMS dispersion among the different objects). The total density is inferred from the circularspeed, $v_{c}$, measured in the $21-\mathrm{cm}$ hydrogen line which, for axisymmetric systems, is related to $v_{c}$ as (e.g., de Blok et al. 2001):

$\rho(r)=\frac{1}{4 \pi G}\left[\frac{v_{c}}{r}\right]^{2}\left[1+2 \frac{\mathrm{d} \log v_{c}}{\mathrm{~d} \log r}\right]$,

were $G$ is the gravitational constant. The scatter of the 26 density profiles gets largely reduced when each individual profile is normalized to the radius and density where $\mathrm{d} \log v_{c} / \mathrm{d} \log r=0.3$, denoted as $r_{0.3}$ and $\rho_{0.3}$, respectively (Oh et al. 2015). In addition to reducing the observational scatter, this normalization makes 
the comparison with polytropes parameter-free. The density $\rho(r)$ consistent with a polytrope of index $m$ (Eq. (2)) depends on two parameters $\rho(0)$ and $b$. Using Eqs. (3) and (5), it can be shown that

$\rho\left(x r_{0.3}\right) / \rho_{0.3}=\psi^{m}\left(x s_{0.3}\right) / \psi^{m}\left(s_{0.3}\right)$,

where $x=r / r_{0.3}$ and $s_{0.3}$ is the value for $r_{0.3}$ obtained from $\psi(s)$. The right-hand side of Eq. (6) does not depend on $\rho(0)$ or $b$, indicating that the same happens with the normalized density (the left-hand side of the equation) which, consequently, has no freedom in Fig. 2. Thus, the agreement between the observed and the predicted cores is particularly revealing, suggesting a true connection between polytropes and the inner structure of dwarf galaxies.

\section{Conclusions}

In this work, we show that the polytropes, resulting from the principle of maximum Tsallis entropy, reproduce without any tuning the cores observed in the matter distribution of dwarf galaxies. The genesis of these cores is currently interpreted as being driven by the interplay between baryons and DM, such that repetitive baryon motions modify the overall gravitational potential and the associated matter distribution (Sect. 1). We note that the two explanations are not in opposition; in fact, they are consistent if the baryon driven motions simply shorten the timescale needed to thermalize the global gravitational potential into a polytrope.

Our study is focused on the central regions of the galaxies, but polytropes also work well in the outskirts (e.g., Saxton \& Ferreras 2010). The outer parts are fully dominated by DM and it has repeatedly been shown that polytropes can be fit with Einasto profiles (e.g., Zavala et al. 2006; Salvador-Solé et al. 2012), which fit well the outer parts of the DM profiles found in cosmological numerical simulations (e.g., Navarro et al. 2004; Merritt et al. 2005; Calvo et al. 2009). In support of this, Frigerio Martins et al. (2015) employ the maximum Tsallis entropy formalism to fit the radial dependence of $v_{c}$ in 24 galaxies with $8 \leq \log \left(M_{\star} / M_{\odot}\right) \leq$ 11. The two variables $v_{c}(r)$ and $\rho(r)$ are interchangeable (Eq. (5)), so that the goodness of the fit at all radial distances also applies to $\rho(r)$ - although Frigerio Martins et al. do not specifically consider the cores we cover in this work.

The association between dwarf galaxies and maximum Tsallis entropy opens up the possibility of using the wellproven tool-kit of statistical mechanics to understand them (Padmanabhan 2008; Pontzen \& Governato 2013; Saxton 2013). Identifying galaxies with polytropes has a number of additional implications. Accurate mass profiles are needed in planning and interpreting the astrophysical experiments for disclosing the nature of DM. The DM annihilation cross-sections depend on halo shape (e.g., Zhao et al. 2018) and precise DM profiles, along with their time evolution, should help us to distinguish between cold, warm, or self-interacting DM (e.g., Weinberg et al. 2015; Ludlow et al. 2016). The suite of mass models currently used in gravitational lensing studies does not include polytropes (e.g., Keeton 2001), but subtle details in the mass model are critically important when precise magnifications are needed, or when lensing is used to derive cosmological parameters (Knudson et al. 2001; Elíasdóttir \& Möller 2007).

The ability of polytropes to reproduce observed galaxy properties also has an impact on the side of statistical mechanics. A comparison with the cosmic evolution of astronomical objects will shed new light on whether the $S_{q}$ entropies, in addition to providing $\mathrm{H}$-funtionals that are capable of selecting particular steady-state solutions of the Vlasov equation (Chavanis \& Sire 2005), would also have a deeper thermodynamical significance for self-gravitating systems.

Acknowledgements. Thanks are due to Jose Diego for discussions on the mass models used in gravitational lensing analysis, to Bruce Elmegreen for help and references on the stability of disk galaxies, and to Arianna Di Cintio for references on the core-cusp problem. J. S. A. acknowledges support from the Spanish Ministry of Economy and Competitiveness (MINECO), projects AYA2016-79724-C4-2-P and PID2019-107408GB-C43 (ESTALLIDOS). IT also acknowledges financial support from the European Union's Horizon 2020 research and innovation programme under Marie Skłodowska-Curie grant agreement No 721463 to the SUNDIAL ITN network, from the State Research Agency (AEI) of the Spanish Ministry of Science, Innovation and Universities (MCIU) and the European Regional Development Fund (FEDER) under the grant with reference AYA2016-77237-C3-1-P, from IAC projects P/300624 and P/300724, financed by the Ministry of Science, Innovation and Universities, through the State Budget and by the Canary Islands Department of Economy, Knowledge and Employment, through the Regional Budget of the Autonomous Community, and from the Fundación BBVA under its 2017 programme of assistance to scientific research groups, for the project Using machine-learning techniques to drag galaxies from the noise in deep imaging.

\section{References}

Binney, J., \& Tremaine, S. 2008, Galactic Dynamics: Second Edition (Princeton, NJ, USA: Princeton University Press)

Blanton, M. R., Hogg, D. W., Bahcall, N. A., et al. 2003, ApJ, 594, 186

Brown, S. T., McCarthy, I. G., Diemer, B., et al. 2020, MNRAS, 495, 4994

Calvo, J., Florido, E., Sánchez, O., et al. 2009, Phys. Stat. Mech. Appl., 388, 2321

Cen, R. 2014, ApJ, 790, L24

Chandrasekhar, S. 1967, An Antroduction to the Study of Stellar Structure (New York: Dover)

Chavanis, P. H., \& Sire, C. 2005, Phys. Stat. Mech. Appl., 356, 419

Colín, P., Avila-Reese, V., \& Valenzuela, O. 2000, ApJ, 542, 622

de Blok, W. J. G., McGaugh, S. S., Bosma, A., \& Rubin, V. C. 2001, ApJ, 552 L23

de Jong, R. S., \& van der Kruit, P. C. 1994, A\&AS, 106, 451

Del Popolo, A., \& Le Delliou, M. 2017, Galaxies, 5, 17

de Vaucouleurs, G. 1948, Ann. Astrophys., 11, 247

Elíasdóttir, Á., \& Möller, O. 2007, JCAP, 2007, 006

Frigerio Martins, C., Lima, J. A. S., \& Chimenti, P. 2015, MNRAS, 449, 3645

Governato, F., Brook, C., Mayer, L., et al. 2010, Nature, 463, 203

Hindmarsh, A. C. 2019, Astrophysics Source Code Library, [record ascl:1905.021]

Keeton, C. R. 2001, ArXiv e-prints [arXiv:astro-ph/0102341]

Knudson, A., Ratnatunga, K. U., \& Griffiths, R. E. 2001, AJ, 122, 103

Lima, J. A. S., \& de Souza, R. E. 2005, Phys. Stat. Mech. Appl., 350, 303

Livadiotis, G., \& McComas, D. J. 2013, Space Sci. Rev., 175, 183

Ludlow, A. D., \& Angulo, R. E. 2017, MNRAS, 465, L84

Ludlow, A. D., Bose, S., Angulo, R. E., et al. 2016, MNRAS, 460, 1214

Merritt, D., Navarro, J. F., Ludlow, A., \& Jenkins, A. 2005, ApJ, 624, L85

Moore, B. 1994, Nature, 370, 629

Navarro, J. F., Hayashi, E., Power, C., et al. 2004, MNRAS, 349, 1039

Nipoti, C. 2015, ApJ, 805, L16

Oh, S.-H., Hunter, D. A., Brinks, E., et al. 2015, AJ, 149, 180

Padmanabhan, T. 2008, ArXiv e-prints [arXiv:0812.2610]

Plastino, A. R., \& Plastino, A. 1993, Phys. Lett. A, 174, 384

Pontzen, A., \& Governato, F. 2013, MNRAS, 430, 121

Salvador-Solé, E., Viñas, J., Manrique, A., \& Serra, S. 2012, MNRAS, 423, 2190

Saxton, C. J. 2013, MNRAS, 430, 1578

Saxton, C. J., \& Ferreras, I. 2010, MNRAS, 405, 77

Silva, J. R. P., Nepomuceno, M. M. F., Soares, B. B., \& de Freitas, D. B. 2013, ApJ, 777, 20

Spergel, D. N., \& Steinhardt, P. J. 2000, Phys. Rev. Lett., 84, 3760

Tsallis, C. 1988, J. Stat. Phys., 52, 479

Tsallis, C. 2009, Introduction to Nonextensive Statistical Mechanics (New York: Springer)

van der Wel, A., Bell, E. F., Häussler, B., et al. 2012, ApJS, 203, 24

Weinberg, D. H., Bullock, J. S., Governato, F., Kuzio de Naray, R., \& Peter, A. H. G. 2015, Proc. Nat. Acad. Sci., 112, 12249

Zavala, J., Núñez, D., Sussman, R. A., Cabral-Rosetti, L. G., \& Matos, T. 2006, JCAP, 2006, 008

Zhao, Y., Bi, X.-J., Yin, P.-F., \& Zhang, X. 2018, Phys. Rev. D, 97, 063013 